doi: $10.26529 /$ cepsj.788

\title{
Editorial
}

\section{Robotisation, Automatisation, the End of Work and the Future of Education}

The present issue of the CEPS Journal invites us to consider the emerging world of the simultaneous functioning of human and artificial intelligence in the field of education.

The articles published in the focus section cover a wide spectrum of considerations related to the triangle human intelligence-artificial intelligenceteaching and learning, a triangle that is itself related to the changes in society that are taking place in parallel.

With the present issue, we as the editorial board of the journal join the already widespread and urgently needed reflective approach to an issue that we as educators should, with dedication and full responsibility towards the future of humanity and education, consider as a constitutive part of education.

We start the focus section with an article entitled Is Machine Learning Real Learning? written by Zdenko Kodelja. The author confronts the ambiguous question of whether machine learning is real learning. It is ambiguous because the term "real learning" can be understood in different ways. Firstly, it can be understood as learning that actually exists and is, as such, opposed to something that only appears to be learning, or is misleadingly called learning although it is something else, something that is different from learning. Secondly, it can be understood as the highest form of human learning, which presupposes that an agent understands what is learned and acquires new knowledge as a justified true belief. Just as there are contradicting conceptions, there are also opposite answers to the question of whether machine learning is real learning. Some experts in the field of machine learning, which is a subset of artificial intelligence, claim that it is in fact learning and not something different from learning, while some others - including philosophers - reject the claim that machine learning is real learning; for them, real learning means the highest form of human learning. The main aim of the author is to present and discuss, very briefly and in a simplifying manner, only some interpretations of human and machine learning, on the one hand, and the problem of real learning, on the other, in order to make it clearer that the answer to the question of whether machine learning is real learning depends on the definition of learning.

The second article, written by Ivan Ivić, addresses another topic pertinent to education: the relationship between Printed and Digital Media: Printed and Digital Textbooks. The aim of this paper is to sketch a framework for comparing 
the educational quality and efficiency of printed and digital textbooks. Considering that digital textbooks are a relatively new phenomenon, we as teachers lack both practical experience with their use and research on their efficiency, which is why all of the results in the present article are, in the author's opinion, "only of a preliminary nature". This is also why the paper focuses on an initial definition of the theoretical framework not only for analysing the nature of digital media, but also for analysing printed and digital textbooks. The broadest theoretical framework consists of Lev Vygotsky's theory on the character of cultural-psychological tools and their influence on the functional organisation of the brain, as well as Marshall McLuhan's theory of media, concerning the general fact of the medium (and not its content). The author claims that theoretical analysis shows that media (printed and digital) have significant impacts on the functional organisation of the brain, which is based on the brain's neuroplasticity. The analysis of digital media also demonstrates that they possess a set of specific features that, despite all of their advantages, carry important risks for the organisation and functioning of the brain and for the process of learning.

In order to offer our readers a concrete example of the preparation of future educators for the shift in their working environment, we have included in the focus the article Digital Making in Educational Projects, written by Alejandra Bosco, Noemí Santiveri and Susanna Tesconi. Digital Making is a conceptualised presentation of an innovative educational experience carried out with students of the Primary Education and Social Education degrees over three consecutive years. The experience highlights digital making as an activity in which students create an object using digital technology, and in doing so learn not only about the functioning of technology, but in parallel also acquire the content and competences of the curriculum. The authors report that the innovative teaching practice presented was carried out as action research in order to improve traditional higher education practices. In this sense, the proposal puts the student at the centre of the process as the author and protagonist of his/her own learning process. The experience is based on students' own interests (they decide what to make based on a given context). Students work in groups and seek what they need to learn in order to meet the challenge, while the teacher supports the process as a facilitator, offering guidance and resources when necessary. The evaluation of the whole process is regulated by a group diary (a shared document online) and an individual diary (a blog) produced by the students. The final evaluation is not only of the printed product; the students also produce a video as storytelling in which they explain how the process evolved from the initial idea to the final impression of the object. This experience of one of the teacher training institutions in Europe was carried out in collaboration 
with the Centres of Digital Fabrication of Barcelona. The results are organised so as to highlight the strengths and weaknesses of using technologies to improve higher education, by offering an approach in which students are at the centre of the whole process.

Yet another aspect of teaching and learning is addressed in the text entitled The Use of Humanoid Robots with Multilingual Interaction Skills in Teaching a Foreign Language: Opportunities, Research Challenges, and Future Research Directions. Authors Ayse Tuna and Gurkan Tuna start their consideration of the specific characteristics of the use of humanoid robots in teaching foreign language with the claim that a humanoid robot can be useful for many educational goals because it does not get tired regardless of how many mistakes the student makes; in addition, it can be equipped with novel teaching techniques and updated with the most current knowledge. According to the authors, robots are more useful as teaching aids than computers, as they can mimic human responses, which is one of the reasons why humans, especially children, prefer robot interaction to other interaction types. Although it may run counter to common sense, the use of humanoid robots leads to a certain type of personal connection with students, which can help overcome issues related to shyness, reluctance, frustration and lack of confidence that may emerge in dealing with a human teacher. Moreover, as humanoid robots can be programmed to know specifically what each individual student needs to learn, they can be quite useful for one-on-one speaking activities. Given the number of questions that remain open, it seems worthwhile to consider "the many possibilities that can be offered by information and communication technology tools, particularly by humanoid robots".

In their article, Don Douglas McMahon and Zachary Walker address Universal Design for Learning (UDL) developed by Rose and Meyer (2002) and updated by CAST (2011). While several researchers have used the UDL framework to inform their decision-making and evaluation process regarding technology interventions for students (Almond et al., 2010; Dolan, Hall, Banerjee, \&Strangman, 2005; Hall, Strangman, \& Meyer, 2003), the authors of the article present it as "one of many potential means of addressing the rapidly changing technology landscape and its impact on education". Their main aim is, they say, to support educators in implementing and adopting emerging technologies so that their students are prepared for a future in which we will all have more robotisation, automatisation, artificial intelligence and immersive learning tools. How are we supposed to respond to the way the future will look and what our classrooms will consequently be like?

While robotisation, automatisation, artificial intelligence and immersive 
learning tools will create new challenges in both the workplace and in education, it is important that we consider how to prepare our students moving forward. Moreover, while the authors understand that certainty is safe and comfortable, they believe that it is equally important to acknowledge that we will never be fully certain of the potential or the challenges of using technology. The implication of innovation is that we will not always know what is going to happen next. The least dangerous assumption is to try to effectively implement new tools in education. The UDL framework and the propositions suggested by the authors are a viable strategy for the effective and informed implementation of these technologies in education. These emerging technologies also present unknown opportunities for new applications supporting inclusion in society and inclusive educational environments. In order to address making emerging technology inclusive, researchers, educators and advocates across many fields need to be informed and inclined to use it with consideration.

The last article in the focus section is entitled Digitalisation in education, allusions and references. In it, Marianna Vivitsou from Helsinki University addresses the metaphor of digitalisation as a phenomenon that became central to education in a period in which budget cuts, privatisation, lay-offs and outsourcing of the labour marked the ethos of twenty-first century competencies. It was precisely this context that put the metaphor in a new light. The aforementioned configuration attributed a mythical fullness to the concept, in the sense that digitalisation goes beyond the limits of a property that needs be developed so that society can successfully deal with contemporary challenges and opportunities.

In this way, digitalisation emerges as a new hegemony in education, with narratives that are more or less directly referential. Less direct references add the element of allusion to the metaphor of digitalisation, in the sense that references can be more implicit/covert or even concealed/hidden. Furthermore, as they combine with abstract terms and concepts, they make the boundaries of the technological and the educational domain blurry and educational discourse vague. In order to examine narratives of digitalisation and how they influence educational discourse, this study aims to discuss and analyse relevant policy documents in relation to research and studies on the integration of digital technologies in classroom settings and the hybrid or blended learning environments that open up. To this end, the study uses thematic analysis and discourse analysis to trace allusions and references and discuss how emergent meanings relate to current and future needs in education generated by digitalisation itself.

In line with the profile of the CEPS Journal, we have included two Varia articles in the issue.

In the article Instructional Leadership Effects on Teachers' Work 
Engagement: Roles of School Culture, Empowerment, and Job Characteristics by Adel Zahed-Babelan, Ghodratollah Koulaei, Mahdi Moeinikia and Ali Rezaei Sharif, "the relations between the principal's instructional leadership, school culture, psychological empowerment, job characteristics, and teachers' work engagement was examined on a sample of 310 elementary school teachers".

In another Varia paper, entitled Competence of Croatian Student-Teachers and Primary School Teachers in the Visual Arts, authors Zlata Tomljenović and Svetlana Novaković demonstrated "the existence of a statistically significant difference between the self-assessment of the importance of specific competences in the visual arts, and the self-assessment of having these competences, both with students of the Croatian faculties of teacher education and primary school teachers".

As usual, the third section of the CEPS Journal brings a book review, in which "In Search of Safety: Confronting Inequality in Women's Imprisonment" by Barbara Owen, James Wells and Joycelyn Pollock is reviewed by Darja Tadić. The book presents a sociological look at the sources of violence and conflicts in women's prisons by focusing on unravelling the structural inequalities that shape conflict contexts in prisons.

Slavko Gaber AND Veronika Tašner 